\title{
Paramedian transparietal approach to a dominant hemisphere intraventricular meningioma: illustrative case
}

\author{
John P. Andrews, MD, Tarun Arora, MD, Philip Theodosopoulos, MD, and Mitchel S. Berger, MD \\ Department of Neurological Surgery, University of California, San Francisco, School of Medicine, San Francisco, California
}

BACKGROUND Meningiomas of the atrium of the lateral ventricle present a unique operative challenge. Parietal transcortical approaches have been described with an oblique approach, but a strictly paramedian approach may offer advantages in a dominant hemisphere atrial meningioma.

OBSERVATIONS The patient presented with several weeks of intermittent headaches. Magnetic resonance imaging (MRI) showed an enhancing intraventricular mass in the atrium of the left lateral ventricle. Three-dimensional reconstructions were created from a preoperative MRI, with 1-mm slices for neuronavigation. Diffusion tensor imaging (DTI) was obtained, and tracts were reconstructed in the patient's three-dimensional brainspace. DTI tractography delineated a paramedian transparietal corridor devoid of functional white matter tracks. The patient was positioned supine, in a semislouch position. A left parietal craniotomy was performed. Neuronavigation identified a gyrus posterior to the sensory cortex, anterior to the optic radiations and medial to superior longitudinal and arcuate fasciculus fiber tracts. The tumor was debulked to allow mobilization to coagulate capsular blood supply. Gross total resection was achieved. The patient was discharged postoperatively on day 3 without neurological deficits.

LESSONS A paramedian transparietal approach to a dominant hemisphere meningioma of the lateral ventricle can be a safe and effective way to resect tumors in this anatomically unique operative corridor.

https://thejns.org/doi/abs/10.3171/CASE21292

KEYWORDS paramedian approach; intraventricular meningioma; atrial meningioma; dominant hemisphere; intraventricular

Intraventricular meningiomas are relatively rare entities, ${ }^{1,2}$ but when they do occur, they are often large and most commonly found in the atria of the lateral ventricle, with a slight predilection for the left side. ${ }^{3}$ Series from as recently as the 1980s showed high morbidity and even mortality associated with intraventricular meningiomas, ${ }^{4}$ with mortalities due mostly to postoperative intraventricular hemorrhage. More recent series, however, show that these tumors can be removed safely with good outcome. ${ }^{5-7}$ Interhemispheric transcallosal, temporal transcortical, and lateral parietal transcortical approaches have all been described.

Kempe are Blaylock are credited as the first to describe the interhemispheric transcallosal approach to atrial lesions. ${ }^{8}$ This approach has the advantage of traversing minimal cortex, and thus avoiding optic radiations, arcuate and superior longitudinal fasciculi that encase this area. Interhemispheric approaches are well described in the literature ${ }^{1,3,9}$ but traversing the splenium can cause disconnection syndromes. $^{10}$ There are reports of alexia without agraphia resulting from a transcallosal approach to an intraventricular meningioma in a patient with right homonymous hemianopsia. ${ }^{11} \mathrm{~A}$ right homonymous hemianopsia should be considered a contraindication to a transcallosal approach. ${ }^{12}$ The transtemporal approach is another option to reach the atrium. One of the advantages touted in favor of such an approach is the relatively early access to choroidal vascular supply of the tumor, if the supplying arteries are not medially displaced by the mass. ${ }^{5}$ This approach is less desirable in the face of a dominant hemisphere tumor because of the risk of posterior temporal cortex and eloquent white matter tracks draped over this operative corridor, as well as optic radiations. ${ }^{6}$ In addition, tumors may displace vasculature to a degree that makes it difficult to generalize the claim that this approach is uniformly advantageous from a vascular supply point of view.

ABBREVIATIONS $3 \mathrm{D}=$ three dimensional; $\mathrm{AF}=$ arcuate fasciculus; $\mathrm{DTI}=$ diffusion tensor imaging; $\mathrm{MRI}=$ magnetic resonance imaging; $\mathrm{SLF}=$ superior longitudinal fasciculus.

INCLUDE WHEN CITING Published August 16, 2021; DOI: 10.3171/CASE21292.

SUBMITTED May 10, 2021. ACCEPTED June 7, 2021.

(C) 2021 The authors, CC BY-NC-ND 4.0 (http://creativecommons.org/licenses/by-nc-nd/4.0/). 

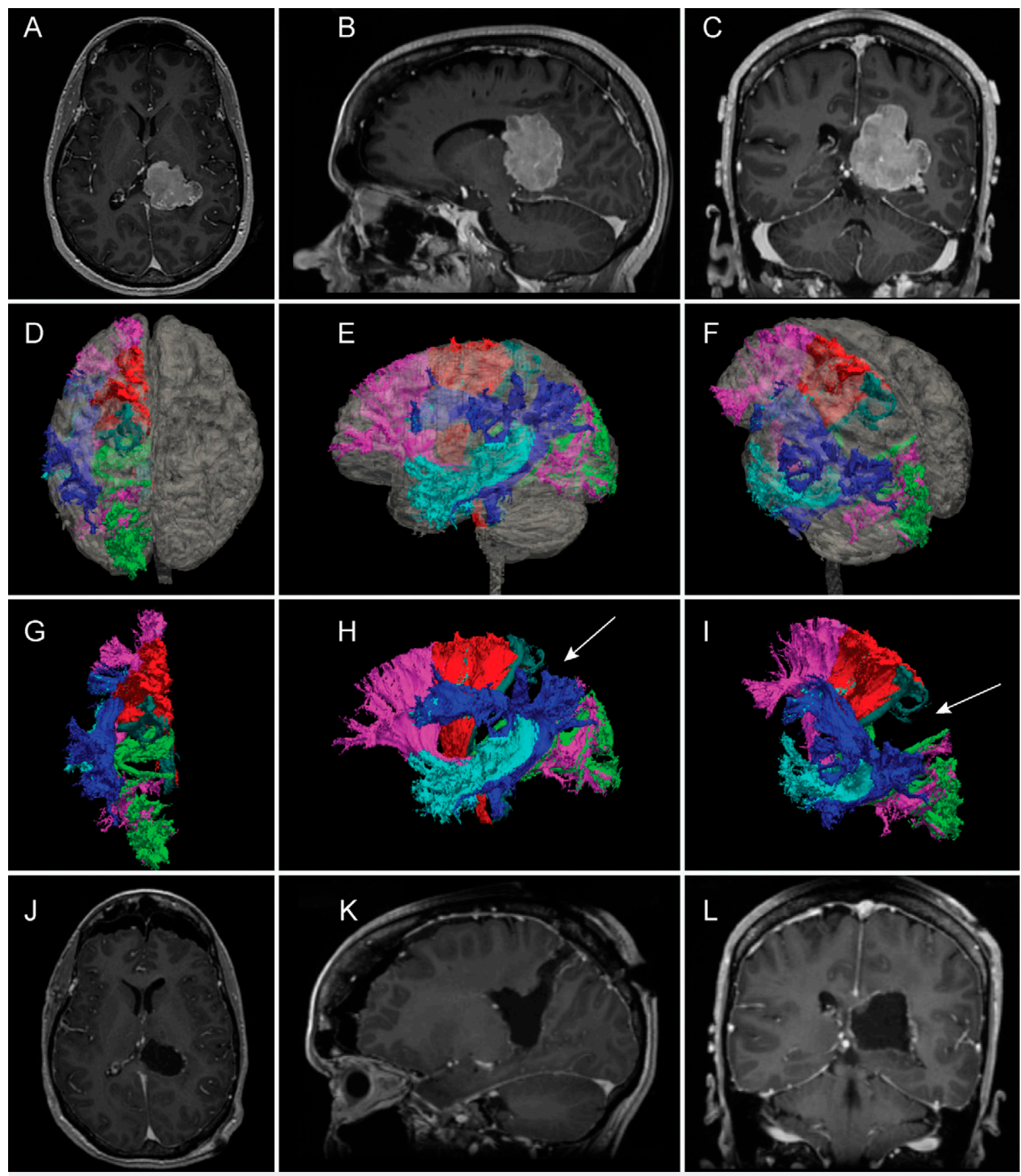

FIG. 1. Preoperative imaging, tractography, and postoperative imaging. A-C: Preoperative T1-weighted, gadolinium-enhanced MRI showing axial (A), sagittal (B), and coronal (C) views of a dominant hemisphere. Intraventricular meningioma. DTI tractography 3D reconstructions of major white matter tracts are depicted in D-I. Superior $(\mathbf{D})$, left lateral $(\mathbf{E})$, and oblique left superior $(\mathbf{F})$ views of a transparent reconstruction of the patient's MRI superimposed. G-I show the same views of the white matter tracts without the brain MRI superimposed. Colors of tracts depicted are as follows: inferior frontal occipital fasciculus, pink; motor tracts, red; sensory tracts, dark green; optic radiations, lime green; SLF, dark blue; AF, light blue. J-L show the patient's postoperative MRI after a transparietal paramedian approach to achieve gross total resection.

The transparietal approach is another option for reaching the atrium of the lateral ventricle. The way this approach appears in the literature often depicts an oblique trajectory to the lateral atrium. ${ }^{1-3,5}$ While it is recognized as a route to avoid optic radiations, late access to blood supply of the tumor is often brought up as a disadvantage of this approach, as well as the possibility of a Gerstmann syndrome. ${ }^{5}$
We describe here a paramedian variation of the transparietal approach. The approach described minimizes danger to the eloquent white matter tracks in the region and allows for both medial and lateral mobilization of the tumor for access blood supply, while the angle of the approach facilitates orientation relative to critical anatomy. 
A

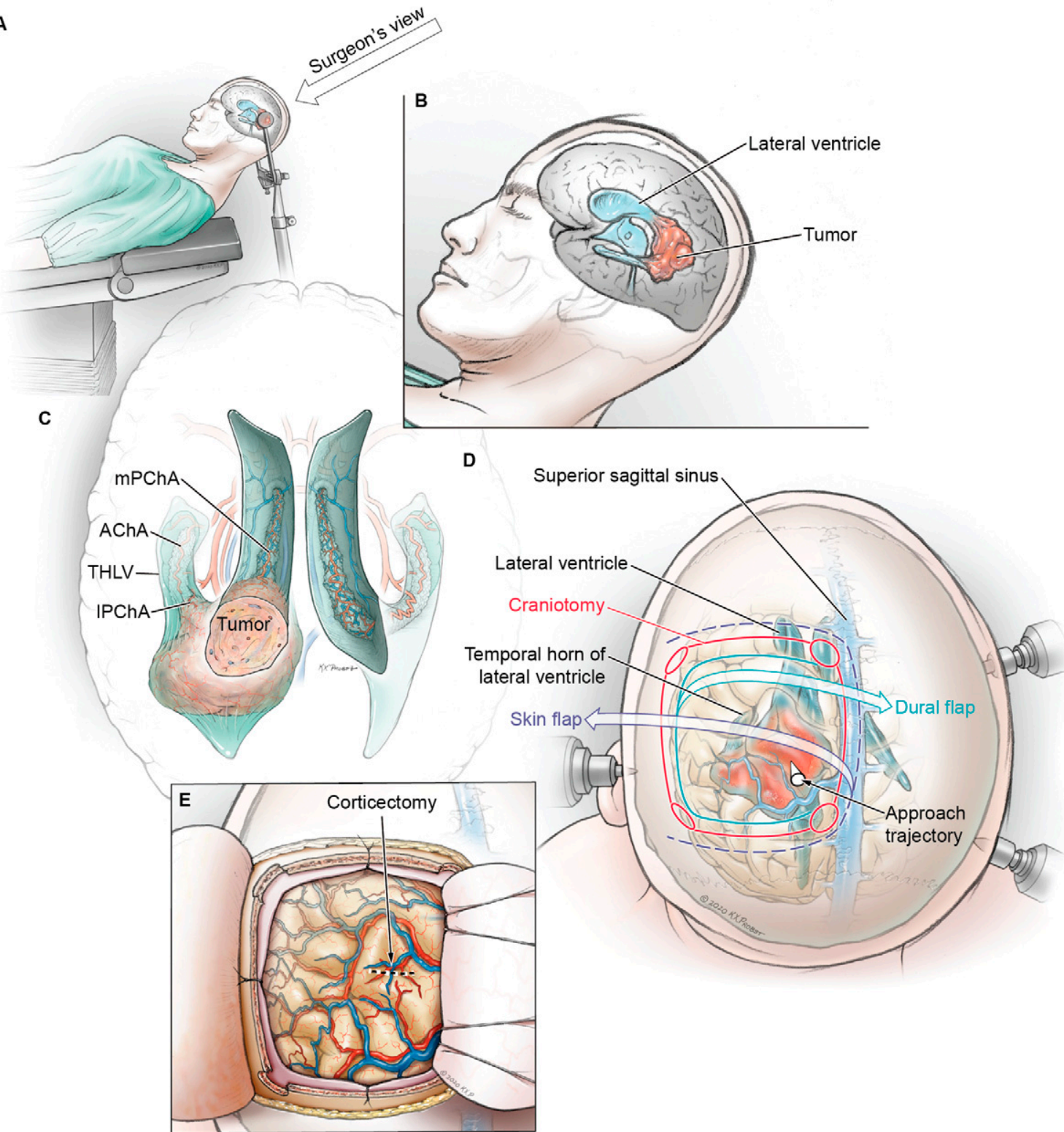

FIG. 2. Positioning and craniotomy. A: Patient positioning supine, in a semislouch position with slight chin tuck. B: Close-up of the head positioning and location of the tumor $(r e d)$ in the ventricular atrium. C: Diagram of the tumor within the ventricular system. D: Surgeon's view of the craniotomy, with the skin incision (dashed blue line), craniotomy (red), and dural opening (turquoise) relative to the underlying tumor, depicted in red, within the ventricular system (turquoise) beneath a transparent cortex, just lateral to the sagittal sinus. E: View of the cortex with the bone flap removed, dura reflected medially and the cortex exposed. $\mathrm{AChA}=$ anterior choroidal artery; IPChA = lateral posterior choroidal artery; $\mathrm{MPChA}=$ medial posterior choroidal artery; THLV = temporal horn of the lateral ventricle.

\section{Illustrative Case}

A 54-year-old female presented with several weeks of intermittent headaches. Preoperative magnetic resonance imaging (MRI) was acquired with and without contrast, with 1-mm slices for threedimensional (3D) neuronavigation. Diffusion tensor imaging (DTI) was obtained, and tracks were reconstructed in the 3D brainspace of the patient's MRI. The 3D coordinate file was reconstructed for intraoperative neuronavigation. MRI showed an enhancing intraventricular mass depicted in Fig. 1. Figure $1 \mathrm{~A}-\mathrm{C}$ shows a T1 MRI with gadolinium contrast, revealing the well-circumscribed, enhancing lesion located in the atrium of the left lateral ventricle. Several factors impact the approach to a lesion such as this, in particular, the white matter pathways surrounding the area. Preoperative diffusor tract imaging (DTI) imaging allows for a nuanced consideration of the white matter tracks in the area. Figure 1D-I shows the results of DTI white matter reconstructions in the area. While transtemporal approaches have been described for lesions in the atria of the lateral ventricles, the reconstructions in Fig. 1 demonstrate the dense arcuate fasciculus (AF) and superior 

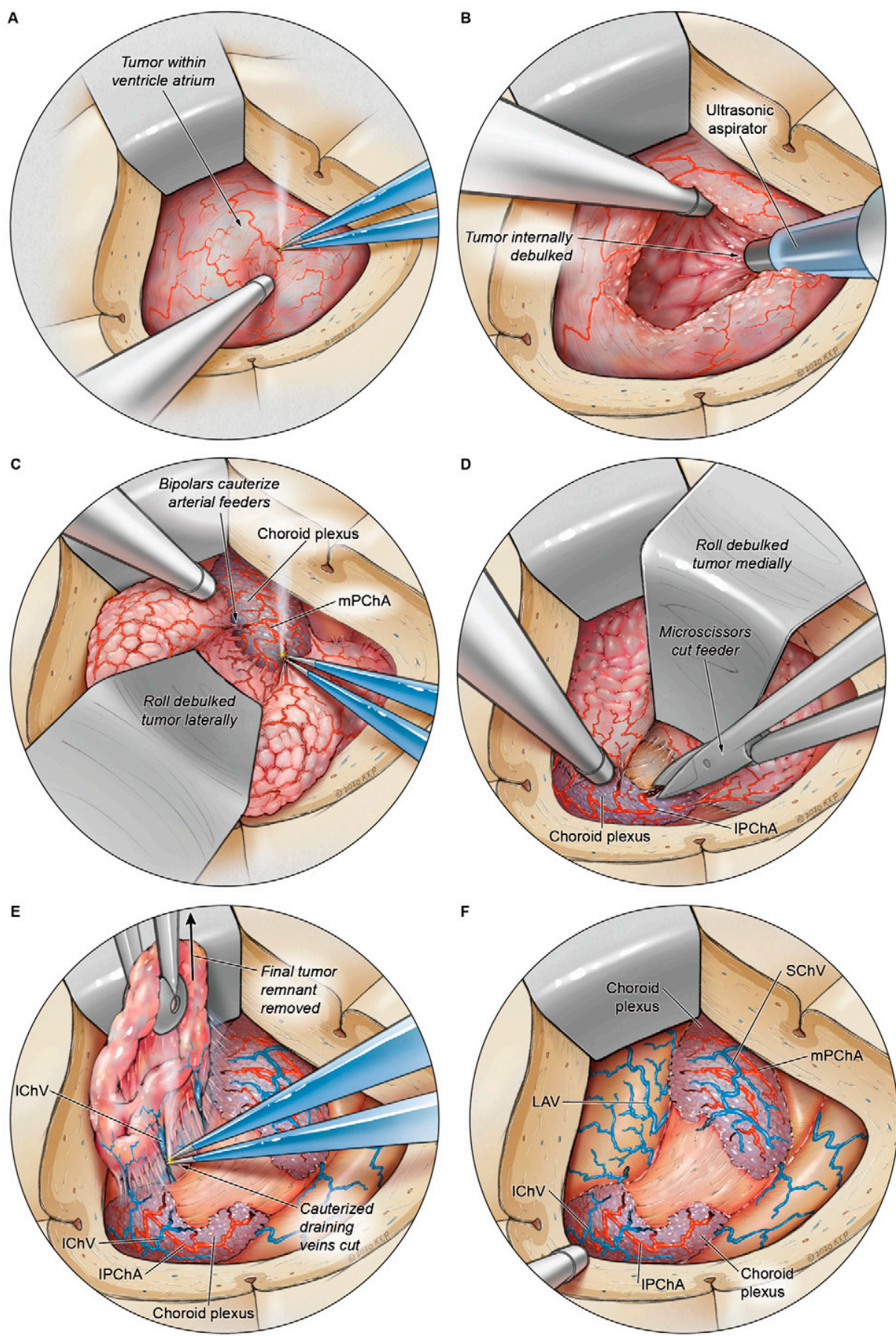

FIG. 3. Tumor mobilization and access to vascular supply. A and B: Tumor resection depicted in the steps of initial tumor view (A), followed by initial debulking (B). C and D: Lateral mobilization for access to medial posterior choroidal feeders (C) and medial mobilization for access to lateral posterior choroidal blood supply (D). D and E: Ligation of remaining draining vascular pedicles $(\mathrm{D})$ prior to removal of the debulked tumor $(\mathrm{E})$. $\mathrm{F}$ : The cavity in the atrium of the lateral ventricle following tumor removal. $\mathrm{AChA}=$ anterior choroidal artery; $\mathrm{IPChA}=$ lateral posterior choroidal artery; LAV = lateral atrial vein; $\mathrm{mPChA}$ = medial posterior choroidal artery; SChV = superior choroidal vein.

longitudinal fasciculus (SLF) blocking an approach through the temporal lobe. At the bottom of Fig. $1 \mathrm{~J}-\mathrm{L}$ is the postoperative MRI showing gross total resection through a transparietal paramedian operative approach.
The transparietal approach presents an opportunity to avoid the white matter tracks (white arrows in Fig. 1G-I). Previous studies have described a transparietal approach but use an oblique trajectory. ${ }^{1-3}$ As shown in Fig. 1D-I, the transcortical, transparietal, and 
paramedian corridor is bounded anteriorly by sensory tracks (dark green), posteriorly by optic radiations (lime green), inferiorly by frontal occipital fasciculus (pink), and laterally by SLF (dark blue) and AF (sky blue). The absence of obvious eloquent white matter tracks medially in Fig. 1 suggests that a paramedian trajectory may give the safest route from a functional perspective.

In order to achieve a paramedian approach to this atrial tumor, the patient was put in a semislouch position, as depicted in Fig. 2, with chin tucked and no head turn (Fig. 2A and B). This aligns the trajectory with the surgeon's line of sight. Figure $2 \mathrm{C}$ diagrams the incision and relation of the craniotomy to the sagittal sinus. The sagittal sinus presents an important medial structure to protect during the paramedian approach. In addition to the sinus itself, large veins draining into the sinus should be considered and avoided. Postcontrast T1 MRI or venography can be used to study the venous anatomy preoperatively.

An inverted U-shaped incision abutting the midline was used, as shown in Fig. 2D. Burr holes are used at each corner of a craniotomy that approaches but does not cross over midline. Most important in this aspect are the two burr holes nearest to midline, which allow for dissection of the dura away from the bone flap at this critical medial portion that is closest to the superior sagittal sinus. With careful dissection and appropriate attention, a bone flap can be turned safely with a footplate.

The dura was opened in a C-shape and flapped toward the superior sagittal sinus (Fig. 2D, turquoise line). Figure 2E then shows the corticectomy made perpendicular to the sagittal sinus. Referring back to the trajectory indicated by white arrows in Fig. $1 \mathrm{H}$ and $\mathrm{I}$, this approach avoids white matter tracks in the area and lead directly to the tumor. In order to guide the surgeon to the tumor in the atrium, neuronavigation is a helpful adjunct, particularly in the sagittal plane. Careful positioning can prepare the surgeon for a very direct, line-ofsight trajectory. In the medial-lateral plane, having the patient with no head tilt will aid the trajectory's anatomical path that parallels the superficial to deep trajectory of the falx and interhemispheric fissure. Measuring the depth of the trajectory also helps orient the surgeon as to when to expect arriving at the ventricle.

Fig. 3 depicts the stepwise removal of the tumor. The blood supply to meningiomas of the atria typically arises from anterior choroidal and posterior choroidal feeders. These will not be initially visible upon entry into the ventricle. As depicted in Fig. $3 A$ and $B$, the tumor must first be internally debulked, for which we used an ultrasonic aspirator. Once the tumor is cored out, the tumor capsule can be mobilized to access vascular supply, as shown in Fig. 3C-F.

The tumor is rolled laterally to expose any choroidal feeders at the deep inferior-medial capsule. These vessels are coagulated with bipolar forceps and incised with microscissors to free the tumor capsule for further mobilization. Subsequently, the tumor is rolled medially to access the anterolateral inferior aspect of the capsule where the anterior choroidal feeders enter. Once these and the tumors' draining veins are coagulated and cut, the tumor should be free to mobilize. The tumor can then be debulked within the ventricle and removed piecemeal. The dura is then closed in water-tight fashion. The postoperative MRI in Fig. 1J-L shows gross total resection. The patient remained without neurological deficits after the surgery and was ultimately discharged home on postoperative day 3 .

\section{Discussion}

Meningiomas arising in the atria of the lateral ventricles present a unique operative challenge. They are deep-seated structures, surrounded by eloquent white matter tracks, and are typically large when they present. We describe a paramedian transparietal approach to an atrial meningioma of the dominant hemisphere. This approach allows for preservation of clinically significant white matter tracks as planned out using preoperative DTI tractography. Prior descriptions of transparietal approaches to the atria have used a more lateral to medial oblique angle to traverse parietal cortex. . $^{1,3,6}$ While such an approach distances the surgeon from the sagittal sinus medially, it can put critical language pathways at risk, namely the SLF and arcuate fasciculus AF, as seen on the preoperative DTI imaging presented in Fig. 1. The paramedian approach minimizes risk to functional language, motor, and visual pathways.

The vascular supply is positioned at the deep edge of the tumor rather than at the entry. To access the vasculature, the tumor must be rolled. This requires some initial debulking in order to carve out space for mobilization of the tumor. Figure 3 shows the lateral and then medial mobilization of the tumor using suction and bipolar forceps. These can be used to creep along the deep aspects of the tumor to incrementally devascularize the choroidal feeders. Once the medial aspect is devascularized, the tumor can then be rolled medially and the technique repeated for the lateral aspect. The vessels are coagulated and then cut with microscissors to free the tumor from its connections. Once the primary feeders have been eliminated, the tumor debulking can occur without significant intratumoral bleeding. Coagulating around the capsule with the bipolar can help shrink the tumor capsule. The tumor may then be removed piecemeal.

\section{Observations}

These tumors can reach impressive size before causing symptoms since they are typically slow-growing tumors. ${ }^{1}$ As with any tumor, different size and anatomical variations can change the options for the best approach. In this case, the tumor had not grown to such a size that any part of the surrounding cortex was flattened or atrophic. When tumors are giant and parts of the cortex are significantly thinned, these may dictate a natural site of corticectomy. The lack of deficits and robust white matter tracks shown on preoperative imaging tells a different story for the present case.

We describe here a transcortical, transparietal paramedian approach to an atrial meningioma. The paramedian trajectory provides an operative corridor to the atrium that is free from eloquent white matter tracks. Preoperative tractography and intraoperative neuronavigation allow the surgeon to identify the safest gyrus for entry. The paramedian trajectory allows for easy orientation relative to key landmarks such as the superior sagittal sinus. While the operative corridor is long, the trajectory is parallel to the falx, which makes anatomical orientation of trajectory intuitive. Oblique transparietal approaches run the risk of imperfect oblique trajectory, which is more difficult to correct once the key orienting ventricular anatomy is identified. In addition, the paramedian approach allows for both medial and lateral mobilization of the tumor for full circumferential access to the anterior and posterior choroidal blood supply.

\section{Lessons}

In conclusion, the vascular, functional, and anatomical considerations of atrial tumors make them a unique neurosurgical entity that demands careful planning. Two reasonable critiques of this approach are the relatively longer transcortical operative corridor that may limit reach at the operative field and the late access to the vascular supply. We manage the former by using a long ultrasonic 
aspirator and an operative microscope. Proper positioning of the patient also offsets difficulties that accompany a deep operative corridor. A retractor can be used judiciously to elevate the superior aspect of the cortex that will begin to fall into the operative field as the tumor is debulked. Care should be taken not to apply excessive retraction as this could also damage nearby white matter tracks.

The paramedian transparietal approach to a dominant hemisphere, atrial meningioma described here allows for an effective way to access tumors in this region to maximize resection while minimizing risk of morbidity.

\section{Acknowledgments}

We would like to thank and acknowledge Kenneth X. Probst for his illustrations for Figs. 2 and 3.

\section{References}

1. Ellenbogen RG. Transcortical surgery for lateral ventricular tumors. Neurosurg Focus. 2001;10(6):E2.

2. D'Angelo VA, Galarza M, Catapano D, Monte V, Bisceglia M, Carosi I. Lateral ventricle tumors: surgical strategies according to tumor origin and development-a series of 72 cases. Neurosurgery. 2005:56(1 Suppl):36-45.

3. McDermott MW. Intraventricular meningiomas. Neurosurg Clin N Am. 2003;14(4):559-569

4. Fornari M, Savoiardo M, Morello G, Solero CL. Meningiomas of the lateral ventricles. Neuroradiological and surgical considerations in 18 cases. J Neurosurg. 1981;54(1):64-74.

5. Juretschke FR, Güresir E, Marquardt G, et al. Trigonal and peritrigonal lesions of the lateral ventricle-surgical considerations and outcome analysis of 20 patients. Neurosurg Rev. 2010;33(4):457-464.

6. Nayar VV, DeMonte F, Yoshor D, Blacklock JB, Sawaya R. Surgical approaches to meningiomas of the lateral ventricles. Clin Neurol Neurosurg. 2010;112(5):400-405.

7. Nanda A, Bir SC, Maiti T, Konar S. Intraventricular meningioma: technical nuances in surgical management. World Neurosurg. 2016;88:526-537.
8. Kempe LG, Blaylock R. Lateral-trigonal intraventricular tumors. A new operative approach. Acta Neurochir (Wien). 1976;35(4): 233-242.

9. Batjer $H$, Samson D. Surgical approaches to trigonal arteriovenous malformations. J Neurosurg. 1987;67(4):511-517.

10. Jun CL, Nutik SL. Surgical approaches to intraventricular meningiomas of the trigone. Neurosurgery. 1985;16(3):416-420.

11. Levin HS, Rose JE. Alexia without agraphia in a musician after transcallosal removal of a left intraventricular meningioma. Neurosurgery. 1979;4(2):168-174.

12. Kawashima M, Li X, Rhoton AL Jr, Ulm AJ, Oka H, Fujii K. Surgical approaches to the atrium of the lateral ventricle: microsurgical anatomy. Surg Neurol. 2006;65(5):436-445.

\section{Disclosures}

The authors report no conflict of interest concerning the materials or methods used in this study or the findings specified in this paper.

\section{Author Contributions}

Conception and design: all authors. Acquisition of data: all authors. Analysis and interpretation of data: Andrews, Berger. Drafting the article: Andrews, Theodosopoulos, Berger. Critically revising the article: all authors. Reviewed submitted version of manuscript: Andrews, Theodosopoulos, Berger. Approved the final version of the manuscript on behalf of all authors: Andrews. Statistical analysis: Berger. Administrative/technical/material support: Arora, Berger. Study supervision: Arora, Berger.

\section{Correspondence}

John P. Andrews: University of California, San Francisco, CA. john. andrews@ucsf.edu. 